\title{
Correlates of Post-Stroke Brain Plasticity, Relationship to Pathophysiological Settings and Implications for Human Proof-of-Concept Studies
}

\author{
Eduardo H. Sanchez-Mendoza and Dirk Matthias Hermann * \\ Department of Neurology, University Hospital Essen, Essen, Germany
}

The promotion of neurological recovery by enhancing neuroplasticity has recently obtained strong attention in the stroke field. Experimental studies support the hypothesis that stroke recovery can be improved by therapeutic interventions that augment neuronal sprouting. However plasticity responses of neurons are highly complex, involving the growth and differentiation of axons, dendrites, dendritic spines and synapses, which depend on the pathophysiological setting and are tightly controlled by extracellular and intracellular signals. Thorough mechanistic insights are needed into how neuronal plasticity is influenced by plasticity-promoting therapies in order not to risk the success

OPEN ACCESS

Edited by: Hansen Wang, University of Toronto, Canada

Reviewed by: Matteo Caleo, National Research Council, Italy Francisca Bronfman, Pontifical Catholic University of Chile,

Chile

Steven Robert Zeiler, Johns Hopkins University, USA

${ }^{*}$ Correspondence:

Dirk Matthias Hermann dirk.hermann@uk-essen.de

Received: 06 April 2016 Accepted: 26 July 2016 Published: 05 August 2016

Citation: Sanchez-Mendoza EH and Hermann DM (2016) Correlates of Post-Stroke Brain Plasticity, Relationship to Pathophysiological Settings and Implications for Human

Proof-of-Concept Studies. Front. Cell. Neurosci. 10:196. doi: 10.3389/fncel.2016.00196 of future clinical proof-of-concept studies.

Keywords: clinical translation, neuronal differentiation, neuronal plasticity, neuronal regeneration, restorative therapy

\section{INTRODUCTION}

Following ischemic stroke, neuronal networks in the vicinity and at distance to the stroke are reorganized. Axons and axon collaterals are enabled to sprout (Reitmeir et al., 2011, 2012) and dendritic and synaptic processes are reshaped (Li et al., 2010; Overman et al., 2012) leading to an overall reorganization of the representational map that mediates the recovery of motor functions in a large number of settings ranging from rodents to primates and humans (Nudo and Milliken, 1996; Nudo et al., 1996; Wang et al., 2010; Overman et al., 2012). These reorganization processes enroll regions of the contralateral hemisphere (Mohajerani et al., 2011; Reitmeir et al., 2011) and have been shown to persist at least for several months post-stroke (Rossini et al., 2003; Sawaki et al., 2014). A wide variety of molecules are involved in these plasticity processes, including cell adhesion and guidance molecules, growth inhibitors, neurotransmitters and transport proteins (Bacigaluppi et al., 2009; Li et al., 2010; Sánchez-Mendoza et al., 2010; Reitmeir et al., 2011) that may activate or inactivate highly complex molecular pathways that enhance or inhibit neuronal sprouting and therefore local and remote connections (Li et al., 2010; Hermann and Chopp, 2012). Unfortunately, the natural capacity of the brain to rewire is insufficient. Though a degree of spontaneous sprouting exists (Li et al., 2010; Reitmeir et al., 2011, 2012) and some spontaneous recovery has been reported in humans (Duncan et al., 2000), neurological deficits persist in the large majority of stroke events even following localized or mild ischemic injuries (Hummel et al., 2005).

The intrinsic capacity of brain repair can be efficiently stimulated by exogenous therapeutic interventions, e.g., by physical exercise, delivery of growth factors, cell-based biologicals 
or pharmacological compounds, which in rodent and primate models of stroke were shown to enhance neurological recovery (Bacigaluppi et al., 2009; Reitmeir et al., 2011, 2012; Jaeger et al., 2015; Wang et al., 2016). Neurological recovery in the experimental setting can be defined as regain of lost function of the paretic limb as compared to a baseline defined previous to the stroke, which should not be confused with neurological compensation (Murphy and Corbett, 2009), in which other parts of the limbs (e.g., shoulder or the nonparetic limb) are recruited to complete a task. Neurological recovery and compensation can be discriminated by specific tests that allow the study of the paretic limb in isolation, e.g., pellet withdrawal in rodents or digit testing in primates that measure fine motor skills (Nudo and Milliken, 1996; Biernaskie et al., 2004), and tests measuring overall motor function, such as the rotarod, tight rope or hand grip tests (Doeppner et al., 2014a). There seems to be a critical time window after stroke in which various interventions, such as voluntary motor stimulation, pharmacological treatment or transcranial brain stimulation, can improve neurological recovery (Nudo et al., 1996; Biernaskie et al., 2004; Hummel et al., 2005; Sawaki et al., 2014; Wahl et al., 2014). In contrast to acute neuroprotective therapies, plasticity-promoting therapies have proven efficacy over weeks or even months post-stroke in animal and human studies.

Within this perspective article, we would like to briefly integrate some findings regarding brain remodeling and plasticity after stroke, elucidating: (a) structural surrogates of successful neurological recovery depending on the localization of ischemic lesions; (b) reorganization and plasticity processes of the cellular, subcellular and network level; (c) critical time windows for various therapeutic interventions; and (d) modes for the delivery of biologicals or drugs. We will shortly present (e) selected molecular signals that are likely mediators of plasticity processes, since we believe that understanding these signals is a major hallmark to prevent the failure of treatments in future clinical studies.

\section{PATHOPHYSIOLOGICAL SETTING INFLUENCES NEUROLOGICAL RECOVERY AND BRAIN PLASTICITY}

Ischemic stroke can affect both gray and white matter tissue, which invariably results in diverse patterns of brain injury and stroke recovery. In both cases, successful stroke recovery goes along with parenchymal tissue remodeling, involving: (a) the survival of neurons and axons in the surrounding of the ischemic lesion, which otherwise exhibit delayed degeneration; (b) the promotion of perilesional axonal, dendritic and synaptic plasticity; (c) the outgrowth of axons and axon collaterals at distance to the stroke; and (d) the modulation of astroglial and microglial responses, which may both promote or impede neuronal sprouting, depending on their activation state (Bacigaluppi et al., 2009; Li et al., 2010, 2014; Liu et al., 2010; Reitmeir et al., 2011, 2012). In a cornerstone study, Nudo and Milliken (1996) showed that local neuronal networks adjacent to the lesion can incorporate surviving neurons into their representational space. In this study, the authors described that neurons involved in distal limb movements before the stroke were recruited into networks involved in proximal limb movements after the stroke (Nudo and Milliken, 1996). Early motor training stimulation was found to retain and expand representational maps of the affected limb (Nudo et al., 1996).

Depending on the severity of injury, damage to white matter may result in complete or incomplete fiber tract lesions. While in the case of complete fiber tract injury virtually no neuronal outgrowth is possible over larger distances, incomplete pyramidal tract injury, as induced by transient middle cerebral artery occlusion, does allow for the de novo formation of new terminal axon collaterals distal to the lesion site, as previously shown in studies in which plasticity of the ipsilateral and contralateral pyramidal tract was analyzed (Reitmeir et al., 2011, 2012). The delivery of the growth factors erythropoietin and vascular endothelial growth factor (VEGF) did not further augment the outgrowth of ipsilesional pyramidal tract fibers but induced the sprouting of midline-crossing contralesional pyramidal axon collaterals that accompanied functional neurological recovery (Figure 1; Reitmeir et al., 2011, 2012), showing a priming of the contralateral hemisphere for neuroplasticity responses that could be pharmacologically enhanced.

\section{PROMOTION OF NEUROLOGICAL RECOVERY AND BRAIN PLASTICITY: WHAT TO DO, WHEN AND HOW}

Aspects of timing should be carefully addressed on restorative therapies to take better advantage of the early brain response to stroke. Several studies indicate a time window of about 1 week after the stroke in animal models and of 1 to 3 months in humans, in which the brain is particularly sensitive to the initiation of stimulating therapies (Biernaskie et al., 2004; Murphy and Corbett, 2009; Leasure and Grider, 2010; Zeiler and Krakauer, 2013; Wahl and Schwab, 2014; Dromerick et al., 2015; Ng et al., 2015). Thus, rats exposed to an enriched environment between 7-14 days, but not at 30 days after stroke, showed a sustained recovery of fine motor skills of the paretic limb (Biernaskie et al., 2004). Likewise, continuous training of the paretic limb started 5 days after the stroke lead to improvement of motor function in primates (Nudo et al., 1996). Moreover, mild voluntary exercise started 7 days after stroke improved somatosensory function in aged rats, while treadmill walking enhanced upper paretic limb skill in humans even months post-stroke (Ploughman et al., 2008; Leasure and Grider, 2010). Interestingly, non-invasive transcranial cortical stimulation, which regulates cortical neurotransmission by a non pharmacological approach, improves motor capacities both on acute, subacute and chronic stroke patients (Kang et al., 2016).

The delivery of biologicals or pharmacological agents also induces a time-dependent response of neurological function. Following neural precursor cell (NPC) delivery after middle cerebral artery occlusion in mice, both acute, sub-acute and post-acute intravenous NPC delivery up to 1 month post- 


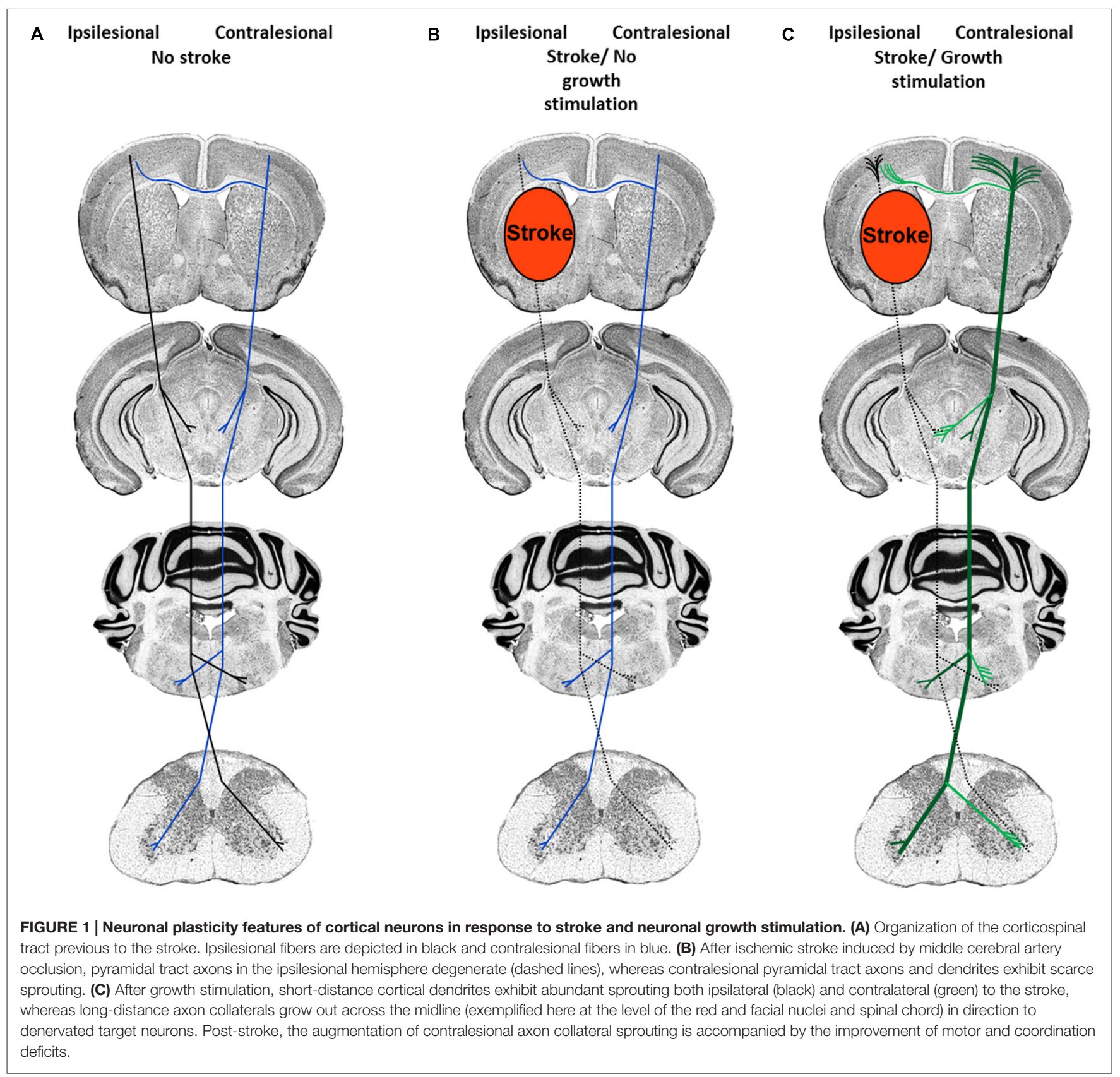

stroke enhanced neurological recovery (Bacigaluppi et al., 2009; Doeppner et al., 2014a,b). While the neurological improvement was independent of the time-point of NPC delivery, the underlying mechanisms strongly varied depending on the timing of NPC grafting. Early delivery of NPCs up to 3 days after stroke potently induced neuroprotection, stabilized blood-brain barrier integrity, decreased brain inflammation and attenuated post-ischemic peripheral immunodepression, whereas postacute NPC delivery at 28 days post-stroke induced more rigorous neuronal differentiation of grafted NPCs associated with enhanced angiogenesis and axonal plasticity (Bacigaluppi et al., 2009; Doeppner et al., 2014b).
Potential pitfalls related to the timing of treatment have been revealed after delivery of neutralizing antibodies against the axonal growth inhibitor NogoA, which, when administered prior to stroke, augmented ischemic injury in a mouse model of transient middle cerebral artery occlusion as a consequence of the early promotion of energy-requiring neuronal growth that resulted in the activation of cell death pathways. Importantly, the post-ischemic delivery of neutralizing NogoA antibodies did not have any injury-promoting effect (Kilic et al., 2010). That appropriate timing of physical and pharmacological therapies is crucial for the therapeutic responses induced was demonstrated in a combination study in which the delivery of neutralizing 
NogoA antibodies was combined with intense motor training. In this study, asynchronous NogoA antibody delivery immediately after stroke, but 2 weeks prior to intense motor training produced a remarkable recovery in fine motor function which was accompanied with contralesional corticospinal tract sprouting, while NogoA antibody delivery in parallel to motor training had no additive effect (Wahl et al., 2014). Time-windows should thoroughly be evaluated in animal studies during the preparation of subsequent clinical trials (Dromerick et al., 2015).

\section{MOLECULAR SIGNALS ASSOCIATED WITH POST-STROKE BRAIN PLASTICITY}

Neuronal sprouting tightly correlates with functional neurological improvement (Hermann and Chopp, 2012). Key molecular signals that control axonal and dendritic sprouting and dendritic spine density are Rho GTPases, phosphatidylinositol-3-kinase/Akt and cyclic nucleotides (Tashiro and Yuste, 2004; Yoshimura et al., 2006; Neukirchen and Bradke, 2011; Averaimo and Nicol, 2014; Spillane and Gallo, 2014). Are axons, dendrites and synapses consistently resuming the same ontogenetic programs that are activated during development (Cramer and Chopp, 2000) or are there also peculiar growth responses that differ between the developing and the stroke brain? In fact, post-stroke plasticity strongly depends on the re-expression of genes that mediate brain connectivity during normal development, which are down-regulated during adulthood under physiological states (Li et al., 2010). Yet, opposite to the developing brain, the post-ischemic brain represents an inflammatory environment that ultimately alters the final outcome of neuronal regrowth (Reitmeir et al., 2011, 2012; Lindau et al., 2014; Barbay et al., 2015). The different pathophysiological setting completely alters patterns of neuronal growth: while during ontogeny long-distance axonal connections are formed within the brain, post-stroke plasticity depends to a strong degree on terminal axon collateral sprouting (Reitmeir et al., 2011, 2012).

Interventions that modulate post-stroke plasticity share a number of common molecular merging points, of which Rho-GTPases deserve particular attention, since they control cytoskeleton remodeling (Leemhuis et al., 2010; Sun et al., 2012; Takeuchi et al., 2015). Rho-GTPases are a large family of proteins of which Rho-A/B, Rac and Cdc42, which have intensively been studied, have been related to axonal and dendritic sprouting in response to growth factor and neurotransmitter exposure. RhoA has an inhibitory effect on neuronal growth, whereas Rac-1 and Cdc42 have growth-promoting activity (Tashiro and Yuste, 2004; Ponimaskin et al., 2007; Leemhuis et al., 2010; Sun et al., 2012). Notably, both erythropoietin and statins, which promote contralesional pyramidal tract plasticity post-stroke and enhance functional neurological recovery in rodents (Reitmeir et al., 2011; Kilic et al., 2014), modulate Rho-GTPase activity. Erythropoietin inhibits Rho-A, ROCK-1 and ROCK-2 after optical nerve crush, promoting axonal growth (Tan et al., 2012), while it promoted axonal and dendritic growth on hippocampal cells through activation of the phosphatidyl-inositol-3-kinase/Akt pathway (Ransome and Turnley, 2008) that promotes microtubule polymerization (Yoshimura et al., 2006). Pravastatin inhibited the activity of RhoA in hippocampal neurons, thus stimulating dendritic and axonal branching. Deactivation of Nogo-66 receptor inhibited Rho-A, thus promoting neuronal growth (McGee and Strittmatter, 2003), while NogoA neutralization inhibited Rho-A and activated Rac1 and Rho-B (Kilic et al., 2010), thus enhancing post-stroke corticospinal tract plasticity (Lindau et al., 2014).

The timing of molecular pathway modulation is essential for stroke recovery. Following neutralizing NogoA antibody delivery, massive overactivation of the small Rho GTPases Rac1 and RhoB was noticed when the antibody was administered prior to stroke, the former of which overactivated the stress kinases p38 and Jun kinase-1/2 (JNK-1/2) thus activating cell death pathways (Kilic et al., 2010). The finding of exacerbated brain injury may not be an exception of the antagonization of a single molecule, i.e., NogoA, which is suggested by the observation that the deletion of the axonal guidance molecule ephrin-B3 enhanced post-ischemic neurogenesis, but at the same time increased infarct size resulting in poor neurological recovery (Doeppner et al., 2011). Accordingly forced paretic limb training caused secondary excitotoxicity that was reduced after administration of the NMDA receptor inhibitor MK801 (Humm et al., 1999). Since glutamate can modulate Rho-GTPase activity (Ponimaskin et al., 2007), uncontrolled glutamatergic transmission mediated by enhanced expression of VGLUT1 and NMDA receptor overactivation could lead to improper sprouting and delayed excitotoxicity (Sánchez-Mendoza et al., 2010). Importantly post-acute delivery of the uncompetitive NMDA receptor inhibitor memantine promoted neurological recovery and contralesional pyramidal tract sprouting (Wang et al., 2016), indicating that rebalancing of the excessive NMDA receptor stimulation at the right time-point indeed enhances post-stroke brain plasticity.

\section{TRANSLATION OF TREATMENTS TO HUMAN STROKE PATIENTS}

Some biologicals and pharmacological drugs have already passed preclinical proof-of-concept studies in animals and are presently undergoing first clinical studies in human stroke patients.

The neuropeptide cocktail cerebrolysin, which was shown to reduce apoptotic neuronal death in vitro (Gutmann et al., 2002) and to have a number of recovery-promoting, neuroprotective and restorative effects after focal cerebral ischemia in rodents, that included the promotion of axonal and dendritic remodeling (Hartbauer et al., 2001; Alcántara-González et al., 2012; Zhang et al., 2013), was recently reported to show beneficial effect on motor recovery when administered together with rehabilitation therapy in a small explorative randomized placebo-controlled study in human stroke patients (Muresanu et al., 2016). The Action Research Arm Test, not a global disability or dependence score, was the primary endpoint of this study. A larger multicenter trial needs to be performed to more definitely evaluate the efficacy of cerebrolysin. 
The antidepressant fluoxetine, which is frequently used for treating post-stroke depressive syndromes, has been found to promote neurogenesis in vitro (Borkowska et al., 2015) and to extend the therapeutic window for brain rewiring after focal cerebral ischemia in mice ( $\mathrm{Ng}$ et al., 2015). In a small randomized placebo-controlled study in human stroke patients, fluoxetine (20 mg per day) promoted motor recovery evaluated by the Fugl-Meyer motor scale over up to 3 months (Chollet et al., 2011). Global disability or dependence, was again not the primary endpoint of this study. A larger sufficiently powered efficacy study is urgently needed. In mice, fluoxetine was efficient in mice only when initiated $24 \mathrm{~h}$, but not 7 days post-stroke, highlighting the relevance of therapeutic windows (Ng et al., 2015).

GSK249320 is an antibody that interferes the interaction of myelin-associated glycoprotein (MAG) and NogoA receptor. GSK249320 has been found to enhance neurite formation in vitro and reduce ischemic infarct size in mice, resulting in enhanced neurological recovery (Irving et al., 2005). Importantly, GSK249320 improved motor function after focal cerebral ischemia in squirrel monkeys (Barbay et al., 2015) and did not reveal any unfavorable effects in a safety study in healthy volunteers (Abila et al., 2013). Larger efficacy studies should be carried out in the future.

The phosphodiesterase inhibitor sildenafil, which increases intracellular concentrations of cGMP, a cyclic nucleotide involved in neuronal plasticity (Averaimo and Nicol, 2014), was shown to enhance the neurological recovery and neuronal plasticity in rat and mouse models of focal cerebral ischemia, stimulating axonal sprouting, neurogenesis, angiogenesis and oligodendrogenesis (Ding et al., 2008). Sildenafil was safe, when administered at a dose of $25 \mathrm{mg}$ per day over 14 days in human subjects (Silver et al., 2009; Chen et al., 2011). A randomized multicenter study is warranted.

The delivery of the inverse agonist for the $\mathrm{GABA}_{\mathrm{A}} \alpha 5$ receptor L655708 was found to enhance neurological recovery after focal cerebral ischemia in mice via mechanisms including the reversal of excessive tonic inhibition of peri-infarct cortex (Clarkson et al., 2010). Following efficacy studies after proximal transient

\section{REFERENCES}

Abila, B., Cunningham, E., and Simeoni, M. (2013). First-time-in-human study with GSK249320, a myelin-associated glycoprotein inhibitor, in healthy volunteers. Clin. Pharmacol. Ther. 93, 163-169. doi: 10.1038/clpt.2012.227

Alcántara-González, F., Mendoza-Perez, C. R., Zaragoza, N., Juarez, I., ArroyoGarcía, L. E., Gamboa, C., et al. (2012). Combined administration of cerebrolysin and donepezil induces plastic changes in prefrontal cortex in aged mice. Synapse 66, 938-949. doi: 10.1002/syn.21588

Averaimo, S., and Nicol, X. (2014). Intermingled cAMP, cGMP and calcium spatiotemporal dynamics in developing neuronal circuits. Front. Cell. Neurosci. 8:376. doi: $10.3389 /$ fncel.2014.00376

Bacigaluppi, M., Pluchino, S., Peruzzotti-Jametti, L., Kilic, E., Kilic, U., Salani, G., et al. (2009). Delayed post-ischaemic neuroprotection following systemic neural stem cell transplantation involves multiple mechanisms. Brain 132, 2239-2251. doi: 10.1093/brain/awp174

Barbay, S., Plautz, E. J., Zoubina, E., Frost, S. B., Cramer, S. C., and Nudo, R. J. (2015). Effects of postinfarct myelin-associated glycoprotein antibody treatment on motor recovery and motor map plasticity in squirrel monkeys. Stroke 46, 1620-1625. doi: 10.1161/strokeaha.114.008088 focal cerebral ischemia in mice and peripheral permanent focal cerebral ischemia in rats confirming enhanced neurological recovery using another $\mathrm{GABA}_{\mathrm{A}} \alpha 5$ receptor antagonist, S44819, proof-of-concept studies have been done in healthy humans, which confirmed the safety of S44819 over a wide dose range. A randomized multicenter study will be initiated in autumn 2016.

\section{SUMMARY AND FUTURE CHALLENGES}

In view of the robust evidence that neurological recovery may be stimulated by therapeutic interventions that enhance neuronal plasticity, there is considerable hope that we may soon become able to use those therapies for enhancing neurological recovery in stroke patients. Stringent proof-of-concept studies are needed that comprise clear-defined "Go" and "No Go" decisions. For a proper idea of drug actions, morphological and molecular insights are required in the preparation of clinical trials that identify substrates of neuronal plasticity, which are subsequently targeted in stroke patients. In case of plasticity-promoting therapies, only well-defined proof-of-concept studies that define: (1) which type of tissue shall be targeted (previously ischemic, peri-lesional or lesion-remote tissue?), and (2) which structural features of neurons are influenced (neuronal survival, axon/axon collateral growth, dendrite/synapse formation?; see also Figure 1) and which is the most appropriate therapeutic window, will allow us to bring plasticity-promoting therapies into clinics.

\section{AUTHOR CONTRIBUTIONS}

Both the authors (EHS-M and DMH) wrote the article. All authors listed, have made substantial, direct and intellectual contribution to the work, and approved it for publication.

\section{FUNDING}

This work was supported by the German Research Council (HE3173/3-1).

Biernaskie, J., Chernenko, G., and Corbett, D. (2004). Efficacy of rehabilitative experience declines with time after focal ischemic brain injury. J. Neurosci. 24 , 1245-1254. doi: 10.1523/JNEUROSCI.3834-03.2004

Borkowska, P., Kowalska, J., Fila-Danilow, A., Bielecka, A. M., PaulSamojedny, M., Kowalczyk, M., et al. (2015). Affect of antidepressants on the In vitro differentiation of rat bone marrow mesenchymal stem cells into neuronal cells. Eur. J. Pharm. Sci. 73, 81-87. doi: 10.1016/j.ejps.2015.03.016

Chen, J., Ye, X., Yan, T., Zhang, C., Yang, X. P., Cui, X., et al. (2011). Adverse effects of bone marrow stromal cell treatment of stroke in diabetic rats. Stroke 42, 3551-3558. doi: 10.1161/strokeaha.111.627174

Chollet, F., Tardy, J., Albucher, J. F., Thalamas, C., Berard, E., Lamy, C., et al. (2011). Fluoxetine for motor recovery after acute ischaemic stroke (FLAME): a randomised placebo-controlled trial. Lancet Neurol. 10, 123-130. doi: 10. 1016/S1474-4422(10)70314-8

Clarkson, A. N., Huang, B. S., Macisaac, S. E., Mody, I., and Carmichael, S. T. (2010). Reducing excessive GABA-mediated tonic inhibition promotes functional recovery after stroke. Nature 468, 305-309. doi: 10 1038/nature09511

Cramer, S. C., and Chopp, M. (2000). Recovery recapitulates ontogeny. Trends Neurosci. 23, 265-271. doi: 10.1016/s0166-2236(00)01562-9 
Ding, G., Jiang, Q., Li, L., Zhang, L., Zhang, Z. G., Ledbetter, K. A., et al. (2008). Magnetic resonance imaging investigation of axonal remodeling and angiogenesis after embolicstroke in sildenafil-treated rats. J. Cereb. Blood Flow Metab. 28, 1440-1448. doi: 10.1038/jcbfm.2008.33

Doeppner, T. R., Bretschneider, E., Doehring, M., Segura, I., Sentürk, A., Acker-Palmer, A., et al. (2011). Enhancement of endogenous neurogenesis in ephrin-B3 deficient mice after transient focal cerebral ischemia. Acta Neuropathol. 122, 429-442. doi: 10.1007/s00401-011-0856-5

Doeppner, T. R., Kaltwasser, B., Bähr, M., and Hermann, D. M. (2014a). Effects of neural progenitor cells on post-stroke neurological impairment-a detailed and comprehensive analysis of behavioral tests. Front. Cell. Neurosci. 8:338. doi: 10. 3389/fncel.2014.00338

Doeppner, T. R., Kaltwasser, B., Teli, M. K., Bretschneider, E., Bähr, M., and Hermann, D. M. (2014b). Effects of acute versus post-acute systemic delivery of neural progenitor cells on neurological recovery and brain remodeling after focal cerebral ischemia in mice. Cell Death Dis. 5:e1386. doi: 10.1038/cddis. 2014.359

Dromerick, A. W., Edwardson, M. A., Edwards, D. F., Giannetti, M. L., Barth, J., Brady, K. P., et al. (2015). Critical periods after stroke study: translating animal stroke recovery experiments into a clinical trial. Front. Hum. Neurosci. 9:231. doi: 10.3389/fnhum.2015.00231

Duncan, P. W., Lai, S. M., and Keighley, J. (2000). Defining post-stroke recovery: implications for design and interpretation of drug trials. Neuropharmacology 39, 835-841. doi: 10.1016/s0028-3908(00)00003-4

Gutmann, B., Hutter-Paier, B., Skofitsch, G., Windisch, M., and Gmeinbauer, R. (2002). In vitro models of brain ischemia: the peptidergic drug cerebrolysin protects cultured chick cortical neurons from cell death. Neurotox. Res. 4, 59-65. doi: 10.1080/10298420290007637

Hartbauer, M., Hutter-Paie, B., and Windisch, M. (2001). Effects of cerebrolysin on the outgrowth and protection of processes of cultured brain neurons. J. Neural Transm. (Vienna) 108, 581-592. doi: 10.1007/s007020 170058

Hermann, D. M., and Chopp, M. (2012). Promoting brain remodelling and plasticity for stroke recovery: therapeutic promise and potential pitfalls of clinical translation. Lancet Neurol. 11, 369-380. doi: 10.1016/s14744422(12)70039-x

Humm, J. L., Kozlowski, D. A., Bland, S. T., James, D. C., and Schallert, T. (1999). Use-dependent exaggeration of brain injury: is glutamate involved? Exp. Neurol. 157, 349-358. doi: 10.1006/exnr.1999.7061

Hummel, F., Celnik, P., Giraux, P., Floel, A., Wu, W. H., Gerloff, C., et al. (2005). Effects of non-invasive cortical stimulation on skilled motor function in chronic stroke. Brain 128, 490-499. doi: 10.1093/brain/awh369

Irving, E. A., Vinson, M., Rosin, C., Roberts, J. C., Chapman, D. M., Facci, L., et al. (2005). Identification of neuroprotective properties of anti-MAG antibody: a novel approach for the treatment of stroke? J. Cereb. Blood Flow Metab. 25, 98-107. doi: 10.1038/sj.jcbfm.9600011

Jaeger, H. M., Pehlke, J. R., Kaltwasser, B., Kilic, E., Bähr, M., Hermann, D. M., et al. (2015). The indirect NMDAR inhibitor flupirtine induces sustained post-ischemic recovery, neuroprotection and angioneurogenesis. Oncotarget 6, 14033-14044. doi: 10.18632/oncotarget.4226

Kang, N., Summers, J. J., and Caraugh, J. H. (2016). Non-invasive brain stimulation improves paretic limb force production: a systematic review and meta-analysis. Brain Stimul. doi: 10.1016/j.brs.2016.05.005 [Epub ahead of print].

Kilic, E., Elali, A., Kilic, U., Guo, Z., Ugur, M., Uslu, U., et al. (2010). Role of Nogo-A in neuronal survival in the reperfused ischemic brain. J. Cereb. Blood Flow Metab. 30, 969-984. doi: 10.1038/jcbfm.2009.268

Kilic, E., Reitmeir, R., Kilic, U., Caglayan, A. B., Beker, M. C., Kelestemur, T., et al. (2014). HMG-CoA reductase inhibition promotes neurological recovery, perilesional tissue remodeling and contralesional pyramidal tract plasticity after focal cerebral ischemia. Front. Cell. Neurosci. 8:422. doi: 10.3389/fncel.2014. 00422

Leasure, J. L., and Grider, M. (2010). The effect of mild post-stroke exercise on reactive neurogenesis and recovery of somatosensation in aged rats. Exp. Neurol. 226, 58-67. doi: 10.1016/j.expneurol.2010.08.003

Leemhuis, J., Bouché, E., Frotscher, M., Henle, F., Hein, L., Herz, J., et al. (2010). Reelin signals through apolipoprotein E receptor 2 and Cdc42 to increase growth cone motility and filopodia formation. J. Neurosci. 30, 14759-14772. doi: 10.1523/jneurosci.4036-10.2010
Li, Y., Liu, Z., Xin, H., and Chopp, M. (2014). The role of astrocytes in mediating exogenous cell-based restorative therapy for stroke. Glia 62, 1-16. doi: 10. 1002/glia.22585

Li, S., Overman, J. J., Katsman, D., Kozlov, S. V., Donnelly, C. J., Twiss, J. L., et al. (2010). An age-related sprouting transcriptome provides molecular control of axonal sprouting after stroke. Nat. Neurosci. 13, 1496-1504. doi: 10.1038/nn. 2674

Lindau, N. T., Bänninger, B. J., Gullo, M., Good, N. A., Bachmann, L. C., Starkey, M. L., et al. (2014). Rewiring of the corticospinal tract in the adult rat after unilateral stroke and anti-Nogo-A therapy. Brain 137, 739-756. doi: 10 1093/brain/awt336

Liu, Z., Li, Y., Zhang, Z. G., Cui, X., Cui, Y., Lu, M., et al. (2010). Bone marrow stromal cells enhance inter- and intracortical axonal connections after ischemic stroke in adult rats. J. Cereb. Blood Flow Metab. 30, 1288-1295. doi: 10. $1038 /$ jcbfm. 2010.8

McGee, A. W., and Strittmatter, S. M. (2003). The Nogo-66 receptor: focusing myelin inhibition of axon regeneration. Trends Neurosci. 26, 193-198. doi: 10. 1016/s0166-2236(03)00062-6

Mohajerani, M. H., Aminoltejari, K., and Murphy, T. H. (2011). Targeted ministrokes produce changes in interhemispheric sensory signal processing that are indicative of disinhibition within minutes. Proc. Natl. Acad. Sci. U S A 108, E183-E191. doi: 10.1073/pnas.1101914108

Muresanu, D. F., Heiss, W. D., Hoemberg, V., Bajenaru, O., Popescu, C. D., Vester, J. C., et al. (2016). Cerebrolysin and recovery after stroke (CARS): a randomized, placebo-controlled, double-blind, multicenter trial. Stroke 47, 151-159. doi: 10.1161/strokeaha.116.012979

Murphy, T. H., and Corbett, D. (2009). Plasticity during stroke recovery: from synapse to behaviour. Nat. Rev. Neurosci. 10, 861-872. doi: 10.1038/nrn2735

Neukirchen, D., and Bradke, F. (2011). Neuronal polarization and the cytoskeleton. Semin. Cell Dev. Biol. 22, 825-833. doi: 10.1016/j.semcdb.2011. 08.007

Ng, K. L., Gibson, E. M., Hubbard, R., Yang, J., Caffo, B., O’brien, R. J., et al. (2015). Fluoxetine maintains a state of heightened responsiveness to motor training early after stroke in a mouse model. Stroke 46, 2951-2960. doi: 10. 1161/STROKEAHA.115.010471

Nudo, R. J., and Milliken, G. W. (1996). Reorganization of movement representations in primary motor cortex following focal ischemic infarcts in adult squirrel monkeys. J. Neurophysiol. 75, 2144-2149.

Nudo, R. J., Wise, B. M., Sifuentes, F., and Milliken, G. W. (1996). Neural substrates for the effects of rehabilitative training on motor recovery after ischemic infarct. Science 272, 1791-1794. doi: 10.1126/science.272.5269.1791

Overman, J. J., Clarkson, A. N., Wanner, I. B., Overman, W. T., Eckstein, I., Maguire, J. L., et al. (2012). A role for ephrin-A5 in axonal sprouting, recovery and activity-dependent plasticity after stroke. Proc. Natl. Acad. Sci. U S A 109, E2230-E2239. doi: 10.1073/pnas.1204386109

Ploughman, M., McCarthy, J., Bossé, M., Sullivan, H. J., and Corbett, D. (2008). Does treadmill exercise improve performance of cognitive or upperextremity tasks in people with chronic stroke? A randomized cross-over trial. Arch. Phys. Med. Rehabil. 89, 2041-2047. doi: 10.1016/j.apmr.2008. 05.017

Ponimaskin, E., Voyno-Yasenetskaya, T., Richter, D. W., Schachner, M., and Dityatev, A. (2007). Morphogenic signaling in neurons via neurotransmitter receptors and small GTPases. Mol. Neurobiol. 35, 278-287. doi: 10. 1007/s12035-007-0023-0

Ransome, M. I., and Turnley, A. M. (2008). Erythropoietin promotes axonal growth in a model of neuronal polarization. Mol. Cell. Neurosci. 38, 537-547. doi: 10.1016/j.mcn.2008.05.002

Reitmeir, R., Kilic, E., Kilic, U., Bacigaluppi, M., Elali, A., Salani, G., et al. (2011). Post-acute delivery of erythropoietin induces stroke recovery by promoting perilesional tissue remodelling and contralesional pyramidal tract plasticity. Brain 134, 84-99. doi: 10.1093/brain/awq344

Reitmeir, R., Kilic, E., Reinboth, B. S., Guo, Z., Elali, A., Zechariah, A., et al. (2012). Vascular endothelial growth factor induces contralesional corticobulbar plasticity and functional neurological recovery in the ischemic brain. Acta Neuropathol. 123, 273-284. doi: 10.1007/s00401-011-0914-z

Rossini, P. M., Calautti, C., Pauri, F., and Baron, J. C. (2003). Post-stroke plastic reorganisation in the adult brain. Lancet Neurol. 2, 493-502. doi: 10. 1016/s1474-4422(03)00485-X 
Sánchez-Mendoza, E., Burguete, M. C., Castelló-Ruiz, M., González, M. P., Roncero, C., Salom, J. B., et al. (2010). Transient focal cerebral ischemia significantly alters not only EAATs but also VGLUTs expression in rats: relevance of changes in reactive astroglia. J. Neurochem. 113, 1343-1355. doi: 10.1111/j.1471-4159.2010.06707.x

Sawaki, L., Butler, A. J., Leng, X., Wassenaar, P. A., Mohammad, Y. M., Blanton, S., et al. (2014). Differential patterns of cortical reorganization following constraint-induced movement therapy during early and late period after stroke: a preliminary study. NeuroRehabilitation 35, 415-426. doi: 10. 3233/NRE-141132

Silver, B., McCarthy, S., Lu, M., Mitsias, P., Russman, A. N., Katramados, A., et al. (2009). Sildenafil treatment of subacute ischemic stroke: a safety study at 25mg daily for 2 weeks. J. Stroke Cerebrovasc. Dis. 18, 381-383. doi: 10.1016/j. jstrokecerebrovasdis.2009.01.007

Spillane, M., and Gallo, G. (2014). Involvement of Rho-family GTPases in axon branching. Small GTPases 5:e27974. doi: 10.4161/sgtp.27974

Sun, Y., Lim, Y., Li, F., Liu, S., Lu, J. J., Haberberger, R., et al. (2012). ProBDNF collapses neurite outgrowth of primary neurons by activating RhoA. PLoS One 7:e35883. doi: 10.1371/journal.pone.0035883

Takeuchi, S., Katoh, H., and Negishi, M. (2015). Eph/ephrin reverse signalling induces axonal retraction through RhoA/ROCK pathway. J. Biochem. 158, 245-252. doi: 10.1093/jb/mvv042

Tan, H., Zhong, Y., Shen, X., Cheng, Y., Jiao, Q., and Deng, L. (2012). Erythropoietin promotes axonal regeneration after optic nerve crush in vivo by inhibition of RhoA/ROCK signaling pathway. Neuropharmacology 63, 1182-1190. doi: 10.1016/j.neuropharm.2012.06.037

Tashiro, A., and Yuste, R. (2004). Regulation of dendritic spine motility and stability by Racl and Rho kinase: evidence for two forms of spine motility. Mol. Cell. Neurosci. 26, 429-440. doi: 10.1016/j.mcn.2004.04.001

Wahl, A. S., Omlor, W., Rubio, J. C., Chen, J. L., Zheng, H., Schröter, A., et al. (2014). Neuronal repair. Asynchronous therapy restores motor control by rewiring of the rat corticospinal tract after stroke. Science 344, 1250-1255. doi: $10.1126 /$ science. 1253050
Wahl, A. S., and Schwab, M. E. (2014). Finding an optimal rehabilitation paradigm after stroke: enhancing fiber growth and training of the brain at the right moment. Front. Hum. Neurosci. 8:381. doi: 10.3389/fnhum.2014. 00381

Wang, L., Yu, C., Chen, H., Qin, W., He, Y., Fan, F., et al. (2010). Dynamic functional reorganization of the motor execution network after stroke. Brain 133, 1224-1238. doi: 10.1093/brain/awq043

Wang, Y. C., Sanchez-Mendoza, E. H., Doeppner, T. R., and Hermann, D. M. (2016). Post-acute delivery of memantine promotes post-ischemic neurological recovery, peri-infarct tissue remodeling and contralesional brain plasticity. J. Cereb. Blood Flow Metab. doi: 10.1177/0271678x16648971 [Epub ahead of print].

Yoshimura, T., Arimura, N., and Kaibuchi, K. (2006). Signaling networks in neuronal polarization. J. Neurosci. 26, 10626-10630. doi: 10.1523/jneurosci. 3824-06.2006

Zeiler, S. R., and Krakauer, J. W. (2013). The interaction between training and plasticity in the poststroke brain. Curr. Opin. Neurol. 26, 609-616. doi: 10. 1097/WCO.0000000000000025

Zhang, L., Chopp, M., Meier, D. H., Winter, S., Wang, L., Szalad, A., et al. (2013). Sonic hedgehog signaling pathway mediates cerebrolysinimproved neurological function after stroke. Stroke 44, 1965-1972. doi: 10. 1161/STROKEAHA.111.000831

Conflict of Interest Statement: The authors declare that the research was conducted in the absence of any commercial or financial relationships that could be construed as a potential conflict of interest.

Copyright (C) 2016 Sanchez-Mendoza and Hermann. This is an open-access article distributed under the terms of the Creative Commons Attribution License (CC BY). The use, distribution and reproduction in other forums is permitted, provided the original author(s) or licensor are credited and that the original publication in this journal is cited, in accordance with accepted academic practice. No use, distribution or reproduction is permitted which does not comply with these terms. 